Check for updates

Cite this: RSC Adv., 2018, 8, 8787

\title{
Trapping a moving droplet train by bubble guidance in microfluidic networks $\dagger$
}

\author{
Longxiang Zhang, Zhaomiao Liu, (D) * Yan Pang, Xiang Wang, (D) Mengqi Li \\ and Yanlin Ren
}

Trapping a train of moving droplets into preset positions within a microfluidic device facilitates the longterm observation of biochemical reactions inside the droplets. In this paper, a new bubble-guided trapping method, which can remarkably improve the limited narrow two-phase flow rate range of uniform trapping, was proposed by taking advantage of the unique physical property that bubbles do not coalescence with two-phase fluids and the hydrodynamic characteristic of large flow resistance of bubbles. The flow behaviors of bubble-free and bubble-guided droplet trains were compared and analyzed under the same two-phase flow rates. The experimental results show that the droplets trapped by bubble-free guided trapping exhibit the four trapping modes of sequentially uniform trapping, nonuniform trapping induced by break-up and collision, and failed trapping due to squeezing through, and the droplets exhibit the desired uniform trapping in a relatively small two-phase flow rate range. Compared with bubble-free guided droplets, bubble-guided droplets also show four trapping modes. However, the two-phase flow rate range in which uniform trapping occurs is increased significantly and the uniformity of the trapped droplet array is improved. This investigation is beneficial to enhance the applicability of microfluidic chips for storing droplets in a passive way.

Received 20th December 2017

Accepted 21st February 2018

DOI: 10.1039/c7ra13507f

rsc.li/rsc-advances
The methodology for trapping microdroplets can be either active $^{\mathbf{8 - 1 0}}$ or passive, ${ }^{\mathbf{1 1 - 1 7}}$ but the passive method, which utilizes nothing more than the rational design of the microchannel, is more appreciated. The microfluidic trapping network (MTN) proposed in recent years is a typical passive configuration for trapping droplets, and has been successfully applied in the evaluation of protein crystallization, synthesis of inorganic materials and cell analysis. It shows apparent characteristics of low reagent consumption and short reaction time in some applications.,.$^{\mathbf{3 1 8}, 19}$ The MTN consists of an array of repetitive trapping units in which each unit has a bypass and a trapping channel containing a hydrodynamic trap. The droplets are coupled with each other and exhibit complex nonlinear dynamics during the trapping process. ${ }^{20}$ This intricate hydrodynamic behavior is a complicated function of many parameters, including channel geometry, liquid properties, and flow conditions. $^{21}$

Two passive approaches for storing droplets were described by Boukellal et al., ${ }^{11}$ who also discussed the characteristics of droplet transportation. It is indicated that the droplet flow behavior depends on both the wettability of the channel walls and the droplet speed. Simon et $a .^{\mathbf{1 4}}$ experimentally and numerically depicted that the entrance width of the trap chamber, droplet size and interfacial tension between the two phases have significant effects on the droplet dynamics. The trapping-bypass channel resistance ratio $\left(R_{\mathrm{T}} / R_{\mathrm{B}}\right)$ and the twophase flow rate affect the path selection of the droplets and
College of Mechanical Engineering and Applied Electronics Technology, Beijing University of Technology, Beijing 100124, China. E-mail: lzm@bjut.edu.cn

$\dagger$ Electronic supplementary information (ESI) available. See DOI: 10.1039/c7ra13507f 
the flow behavior. Behavior transitions from the droplets being trapped at low flow rates to breaking up and squeezing at higher flow rates were experimentally demonstrated by Bithi et al. ${ }^{13}$ On the basis of the above investigations, a new method that utilizes coalescence between the moving and the static droplets, and the following droplet break-up, was subsequently proposed to obtain a highly monodisperse array of droplets at prescribed locations. ${ }^{21,22}$

Yet, the existing investigations mainly concentrate on the kinetics of biochemical reactions inside the trapped droplets, ${ }^{12,18,23-25}$ and an understanding of the fundamental mechanism of nonlinear dynamics in the MTN is still lacking. Additionally, the two-phase flow rate range in which sequentially uniform trapping occurs is narrow in the reported studies on trapping the droplet train, which severely limits the applicability of MTN-based microfluidics techniques. If a special medium that has physical properties characterized by noncoalescence with two-phase fluids and the hydrodynamic characteristic of large flow resistance guides the movement of the following droplets, the limited narrow flow rate range can be remarkably improved. It should be noted that an air bubble precisely possesses the physical properties of this sought-after medium. In view of this, a trapping mode diagram for bubblefree guided droplets is presented, and the causes of disordered behaviors are analyzed. Based on the defects of bubblefree guided trapping, a novel method is proposed to improve the performance of trapping devices, and the mechanisms behind the improvement are discussed.

\section{Materials and methods}

\subsection{Microfluidic channel}

There are two ways in which droplets can be trapped into the chamber of a trapping unit of the MTN: direct trapping and indirect trapping. The first droplet of the train goes directly into the trapping branch, which has lower hydrodynamic resistance $\left(R_{\mathrm{T}}\right)$, while the subsequent droplet flows through the bypass since $R_{\mathrm{T}}$ is significantly increased to $R_{\mathrm{T}}>R_{\mathrm{B}}$. Alternatively, the first several droplets select the bypass with lower hydrodynamic resistance $\left(R_{\mathrm{B}}\right)$, while the subsequent droplet selects the other branch due to the increased resistance generated by the first several droplets. For direct trapping, as the droplet is subjected to larger continuous phase pressure, the critical flow rate at which the droplet staying in the chamber transitions to squeezing out is smaller compared with indirect trapping. This has also been confirmed by the existing experimental studies. ${ }^{13}$ Indirect trapping is preferred over direct trapping in wider applications. Hence, indirect trapping is studied in this work.

A schematic of the microfluidic channel used to trap the droplet train is shown in Fig. 1(a) in the present study. All trapping units are based on the simple design principle that $R_{\mathrm{T}}$ $>R_{\mathrm{B}}$, so one or several droplets in the front of the train enter the bypass, blocking the oil flow due to the resistance of the moving droplet, and then a subsequent droplet flows through the trapping arm and might get trapped in the circular chambers. In consideration of the difficulty in channel machining and the convenience of droplet visualization, $R_{\mathrm{T}} / R_{\mathrm{B}}=2.8$ was designed in the present work. Both $R_{\mathrm{B}}$ and $R_{\mathrm{T}}$ were calculated by the analytical solution eqn (1) ${ }^{23}$ derived from the Poiseuille flow of a single phase in a rectangular channel along two branches, where $\mu$ is the viscosity of the liquid, and $l, w$, and $h$ are the length, width, and depth of the channel, respectively. The error originating from bends, widening and narrowing is ignored.

$$
R=\frac{12 \mu L}{h^{3} w}\left[1-0.63 \frac{h}{w}\right]^{-1}, \quad(h<w)
$$

The channel contains a droplet generator, a spacing adjustment unit, and 25 trapping units (numbered in accordance with $\mathrm{S}$ type). An enlarged view of a trapping unit is illustrated in
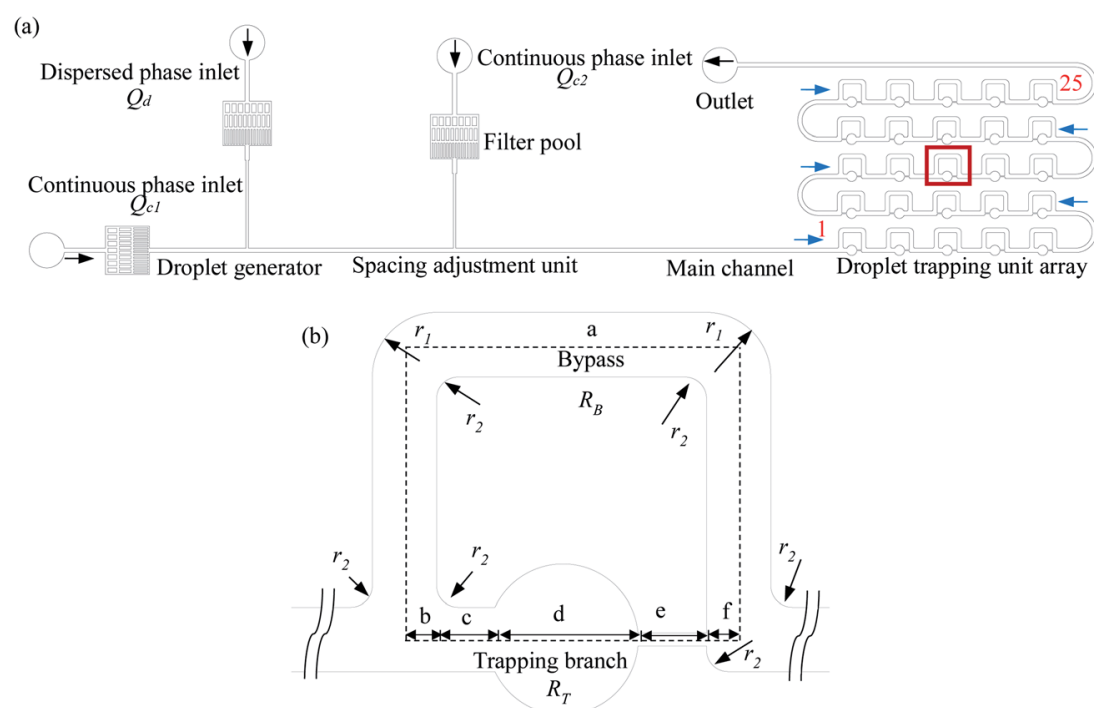

Fig. 1 Schematic diagram of the experimental model, in which (a) is a schematic of the MTN, numbers 1 and 25 represent the trapping unit number, and the blue arrows indicate the droplet movement direction. (b) Represents an enlarged view of the trapping unit from the red rectangle in (a). 
Table 1 Geometric dimensions of the trapping unit (all values are in $\mu \mathrm{m}$ )

\begin{tabular}{|c|c|c|c|c|c|c|c|c|c|c|c|c|c|c|}
\hline \multicolumn{6}{|c|}{ Length of each segment } & \multicolumn{6}{|c|}{ Width of each segment } & \multirow{2}{*}{$\frac{\text { Depth }}{h}$} & \multicolumn{2}{|c|}{ Arc radius } \\
\hline$l_{\mathrm{a}}$ & $l_{\mathrm{b}}$ & $l_{\mathrm{c}}$ & $l_{\mathrm{d}}$ & $l_{\mathrm{e}}$ & $l_{\mathrm{f}}$ & $w_{\mathrm{a}}$ & $w_{\mathrm{b}}$ & $w_{\mathrm{c}}$ & $w_{\mathrm{d}}$ & $w_{\mathrm{e}}$ & $w_{\mathrm{f}}$ & & $r_{1}$ & $r_{2}$ \\
\hline
\end{tabular}

Table 2 Physical properties of the fluids at room temperature $\left(20^{\circ} \mathrm{C}\right)$

\begin{tabular}{lllll}
\hline & Materials & Density $\rho\left(\mathrm{kg} \mathrm{m}^{-3}\right)$ & Viscosity $\mu(\mathrm{MPa} \mathrm{s})$ & Interfacial tension $\gamma_{1}\left(\mathrm{mN} \mathrm{m}^{-1}\right)$ \\
\hline Continuous phase & Sunflower oil & 922 & 49.2 & 25.3 \\
Dispersed phase & Deionized water & 997.1 & 0.89 & \\
\hline
\end{tabular}

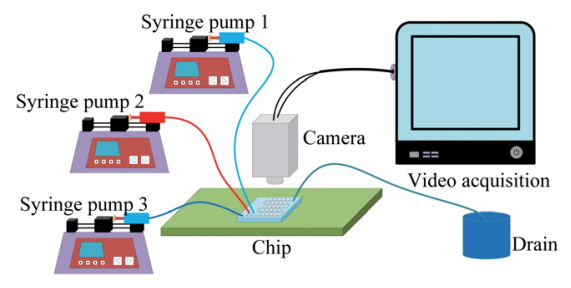

Fig. 2 Schematic diagram of the experimental setup.

Fig. 1(b). The trapping channel is split into five segments: b, c, $\mathrm{d}$, e, and $\mathrm{f}$; these facilitate the calculation of the hydrodynamic resistance and the description of the dimensions. Segment d corresponds to the circular chamber that is rectangular in cross-section. The approximation that a square, the length of one side of which is equal to the diameter of the circle, replaces the chamber in the calculation is applied. $R_{\mathrm{T}}$ is the sum of the flow resistances of the five segments. The error induced by the approximation can be reasonably ignored since the flow resistance of the constriction is a main contributor to $R_{\mathrm{T}}$. The dimensions of the trapping unit are summarized in Table 1 . The device is made of polydimethylsiloxane (PDMS) using standard photolithography techniques. ${ }^{26}$

\subsection{Fluid materials}

Sunflower oil was used as the oil phase due to its slight swelling effect on the channel walls. Deionized water was employed as the dispersed phase. The physical properties of the fluids are shown in Table 2, where $\mu$ and $\gamma$ were measured using a rheometer (R/S Plus, Brookfield, USA) and an interfacial tension meter (A201, Solon Information Technology Co. Ltd., China), respectively.

\subsection{Experimental setup}

The experimental setup is schematically illustrated in Fig. 2. Syringe pumps (Harvard Apparatus, Elite 1012, USA) were used to deliver the two-phase liquids into the channel. Three inlet ports were separately connected to $1000 \mu \mathrm{L}$ glass syringes (Hamilton Company, USA) via peek capillary tubes, while the outlet port was connected to the drain. A high-speed camera (Keyence VW600C, Japan) was applied to visualize the transportation of the train at the frame rate of $30 \mathrm{~Hz}$. The oil phase was pumped into the channel a few hours before the experiments to ensure that the walls were sufficiently wetted.

In the experiment, the normalized droplet length $\zeta=l_{\mathrm{d}} / w_{\mathrm{m}}$ ranged from 1.7 to 2.3 , where $l_{\mathrm{d}}$ and $w_{\mathrm{m}}$ are the droplet length and the width of the main channel, respectively. The average droplet velocity $\left(v_{\mathrm{d}}\right)$ varied from $0.19 \mathrm{~mm} \mathrm{~s}^{-1}$ to $2.16 \mathrm{~mm} \mathrm{~s}^{-1}$ in the main channel. The normalized droplet spacing $\lambda=l_{\mathrm{s}} / l_{\mathrm{a}}$ achieved by adjusting $Q_{\mathrm{c} 2}$ was maintained at an approximately constant value of 0.40 , such that hydrodynamic feedback between the droplets in the bypass and the incoming droplets in the main channel was induced, where $l_{\mathrm{s}}$ is the droplet spacing in the main channel. The values of $v_{\mathrm{d}}, l_{\mathrm{s}}$, and $l_{\mathrm{d}}$ were determined using a home-made Matlab program. The trapped droplet volume $\left(V_{\mathrm{d}}\right)$ was calculated by multiplying the height of the channel by the projected area of the droplet. The percentage of standard deviation of $V_{\mathrm{d}}$ divided by the average is equal to the polydispersity of the trapped droplet array after trapping. Each trapping experiment was done two times to calculate the average polydispersity.

\subsection{Generation of the guiding bubble}

The generation of a small guiding bubble is a key step. Learning from the approach of drop release mentioned by Boukellal et al. ${ }^{27}$ all of the trapped drops were washed out by the high flow rate (such as $Q_{\mathrm{c} 1}=200 \mu \mathrm{L} \mathrm{h}^{-1}, Q_{\mathrm{c} 2}=300 \mu \mathrm{L} \mathrm{h}^{-1}$ ) in the oil phase. The connecting capillary tube was pulled out from the water phase inlet and then the two syringe pumps that deliver the oil were turned off. Oil stains on the PDMS chip surface were cleaned with a dustless cloth wetted by alcohol. Air spontaneously enters the channel from the recently opened inlet. The capillary tube that was pulled out is not re-inserted until air fills the punched inlet hole with a diameter of $0.75 \mathrm{~mm}$ and a length of about $2.5 \mathrm{~mm}$. The filling process is monitored by a highspeed camera. The operation takes roughly 5 and a half minutes to form an air column with a length of about $8.8 w$ to 15 $w$ in the main channel. As the whole column is passing through the T-junction of the drop generator, the two syringe pumps 

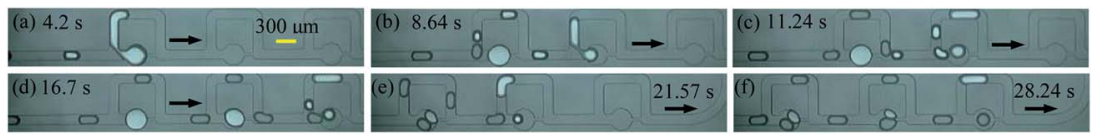

Fig. 3 Experimental results showing generation of the guiding bubble. The arrow indicates the flow direction. (a)-( $f$ ) The generation of the bubble at different times. The time when the long bubble just enters the first junction of the MTN is defined as $0 \mathrm{~s}$. The trapping unit no. of (a) -(d) are 1, 2, and 3. The trapping unit nos of (e) and (f) are 3, 4, and 5.

driving the oil are opened, starting a new capture. Representative results showing the formation of a guiding bubble from the gas column are shown in Fig. 3. It is noted that the bubble column could be cut into a relatively long column and several broken small bubbles at the T-junction of the spacing adjustment unit, and the small bubbles are trapped inside the chambers. When the long bubble enters the first trapping unit, it fragments into a longer portion moving in the bypass and a remnant that fills the whole trap chamber. ${ }^{25,28}$ Subsequently, the longer portion traverses several units (no more than 5) and is gradually dispersed into a guiding bubble with a length of 2.7 $w$ to $2.9 w$. Trapping does not occur in a uniform fashion until the guiding bubble is generated. The procedure for generating the leading bubble is reliable and reproducible. Although bubbles other than drops are stored in the first row of several chambers ( 1 to 4 ), the flow rate range in which $T_{1}$ occurs is significantly broadened by this novel method. It should be noted that the trap chambers occupied by bubbles can be viewed as a waste tank, and enlarging the volume of the wasted chambers and reducing their number can improve the utilization of the MTN.

\section{Results and discussion}

\subsection{Classification of droplet trapping modes}

Trapping modes existing in both cases are classified in this section to compare the trapping effects of the bubble-free and bubble-guided trapping methods. Four trapping modes were exhibited in the MTN: sequentially uniform trapping $\left(\mathrm{T}_{1}\right)$, non- uniform trapping induced by break-up $\left(\mathrm{T}_{2}\right)$, non-uniform trapping induced by collision $\left(\mathrm{T}_{3}\right)$, and failed trapping due to squeezing through $\left(\mathrm{T}_{4}\right)$, as shown in Fig. 4(a)-(d), respectively. Among them, $\mathrm{T}_{1}$ is desired in some applications due to there being no cross-contamination between droplets during the trapping and the acquisition of a highly uniform droplet array. For the bubble-free guided trapping method, the first two droplets in a train choose the bypass in a trapping unit, and then the third droplet is caught by the circular chamber. For the proposed method, the leading bubble flows through the side arm, and the next droplet gets trapped. This mode sequentially occurs in the MTN and is referred to as $T_{1}$.

Long droplets formed by the coalescence of two small droplets in the bypass uncontrollably fragment into two portions at the junction, leaving a broken daughter droplet in the trapping arm. This trapping is identified as $\mathrm{T}_{2}$. When the large droplet moves at high speed, another trapping mode characterized as $\mathrm{T}_{3}$ emerges: a portion of the droplet enters the bypass, while another portion enters the entrance of the trapping channel; before it wholly leaves the junction, the next droplet catches up, and the two neighboring droplets collide. As a result of this collision, the fragment in the trapping arm, or the fragment and its following droplet are trapped. If the differential pressure of the oil phase exerted on the trapped droplet is larger than the Laplace pressure difference between the front and rear caps, the static droplet is pushed out of the chamber. This mode is referred to as $\mathrm{T}_{4}$. It is further demonstrated that one or several modes are observed in a complete trapping process. If one mode occurs more frequently than the others do, this mode is referred to as the dominant

(a): Sequentially uniform trapping $\left(\mathrm{T}_{1}\right)$

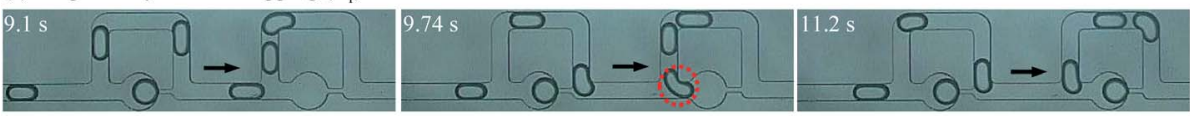

(b): Non-uniform trapping induced by break-up $\left(\mathrm{T}_{2}\right)$

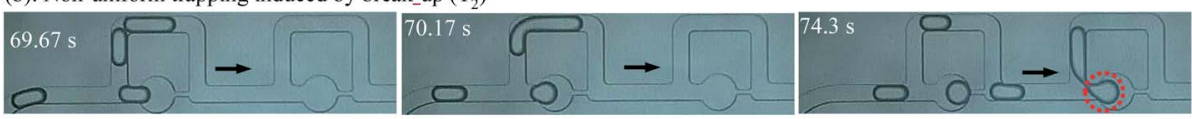

(c): Non-uniform trapping induced by collision $\left(\mathrm{T}_{3}\right)$

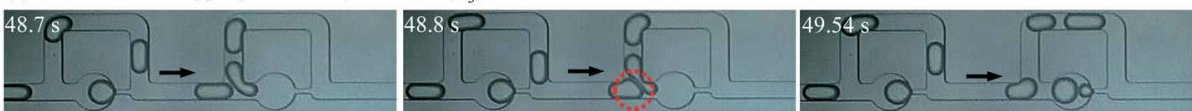

(d): Failed trapping due to squeezing through $\left(\mathrm{T}_{4}\right)$

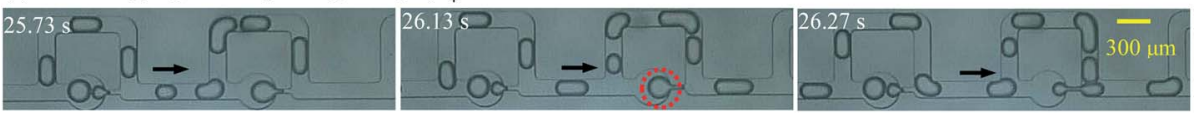

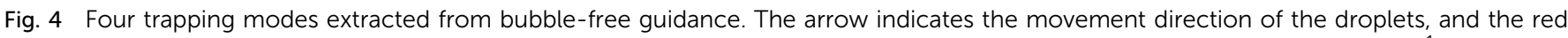

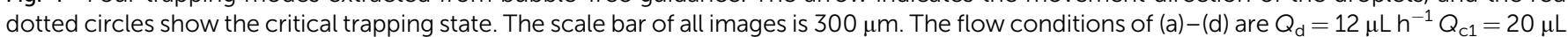

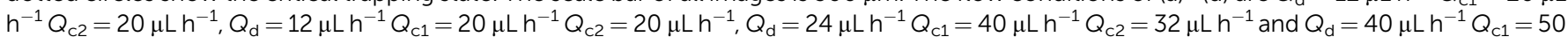
$\mu \mathrm{L} \mathrm{h}{ }^{-1} Q_{c 2}=60 \mu \mathrm{L} \mathrm{h}^{-1}$, respectively. The trapping unit nos of (a)-(d) are 1 and 2, 11 and 12,19 and 20 , and 11 and 12 , respectively. 
(a)

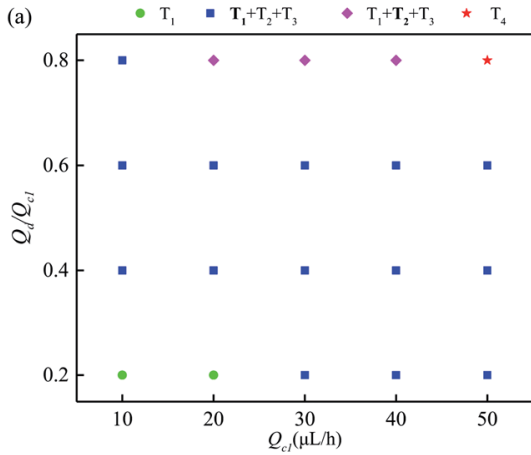

(b)

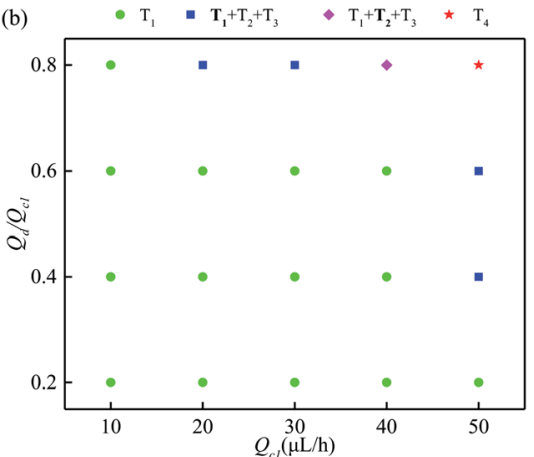

Fig. 5 Trapping mode diagram for bubble-free and bubble guidance (bold indicates the dominant mode); (a) is bubble-free guided trapping and (b) is bubble-guided trapping.

mode. For these trapping modes, there are similarities and differences compared with those of Bithi et al. ${ }^{21}$ Specifically, the definition of $\mathrm{T}_{1}$ is the same as that of $\mathrm{P} 1$ (discrete drop parking). However, $\mathrm{T}_{2}$ is distinguished from P3 (drop parking due to collision-induced break-up) since the long droplet with no surfactant coating in $\mathrm{T}_{2}$ is formed by the coalescence of two small droplets in the bypass, while the long droplet with a surfactant coating in P3 is formed by generation in the upstream $\mathrm{T}$ junction.

\subsection{Comparison of trapping effects by bubble-free and bubble guidance}

A diagram of the trapping modes by bubble-free guidance is illustrated in Fig. 5(a). Small droplets at low speeds were trapped by $\mathrm{T}_{1}$ only in a very narrow range, where the polydispersity was smaller than $14 \%$. The polydispersity depends only on the uniformity of the droplets formed upstream. For droplets of medium speeds and sizes, $T_{1}, T_{2}$ and $T_{3}$, with $T_{1}$ occurring more frequently than the others, were observed in a wide range and the polydispersity ranged from $15 \%$ to $21 \%$. For large droplets moving at high speeds, $\mathrm{T}_{1}, \mathrm{~T}_{2}$ and $\mathrm{T}_{3}$ with $\mathrm{T}_{2}$ dominating were indicated in a small range and the polydispersity was larger than $21 \%$. However, $\mathrm{T}_{4}$ was exhibited when the flow rate exceeded a critical value.

A diagram of the trapping modes of the droplet train by bubble guidance is shown in Fig. 5(b). Compared with bubblefree guided trapping, the region of $\mathrm{T}_{1}$ (green symbol) is broadened significantly. Specifically, the data points of $T_{1}$ are increased from 2 (Fig. 5(a)) to 14. The polydispersity, determined only by the dimensions of the upstream droplets, is less than $14 \%$ at the tested flow rates. Therefore, the flow rate range in which a uniform droplet array is produced is also greatly enhanced by bubble guidance. Taking $Q_{\mathrm{d}}=12 \mu \mathrm{L} \mathrm{h}^{-1}$ $Q_{\mathrm{c} 1}=Q_{\mathrm{c} 2}=20 \mu \mathrm{L} \mathrm{h}^{-1}$ as an example to comparatively show the advancement in detail: $\mathrm{T}_{1}, \mathrm{~T}_{2}$ and $\mathrm{T}_{3}$ occurred 15, 6 and 4 times, respectively, using the bubble-free guided trapping method, and the polydispersity was $19 \%$; all droplets were trapped in the $\mathrm{T}_{1}$ mode (see movie $\mathrm{S} 2, \mathrm{ESI} \dagger$ ) and the polydispersity was $8 \%$ using bubble-guidance.

The fact that there is no cross-contamination between the reaction vessels is one of the most prominent advantages of drop-based microfluidics; however, both $\mathrm{T}_{2}$ and $\mathrm{T}_{3}$ occur in a large range in Fig. 5(a), which not only causes mass transfer between the droplets, but also substantially degrades the uniformity of the trapped droplets. These shortcomings greatly limit the possible biochemical reactions (such as the observation of worm behavior ${ }^{29}$ ), particularly those which have strict requirements for avoiding cross-contamination between reagents, and are sensitive to reagent consumption. It is therefore essential to analyze the principal mechanism of the undesired trapping events in bubble-free guided trapping.

\subsection{Mechanism of undesired non-uniform trapping by bubble-free guidance}

Taking $Q_{\mathrm{d}}=12 \mu \mathrm{L} \mathrm{h}^{-1} Q_{\mathrm{c} 1}=Q_{\mathrm{c} 2}=20 \mu \mathrm{L} \mathrm{h}^{-1}$ as a representative example to demonstrate the mechanism of undesired nonuniform trapping by bubble-free guidance in detail, $\mathrm{T}_{1}, \mathrm{~T}_{2}$,
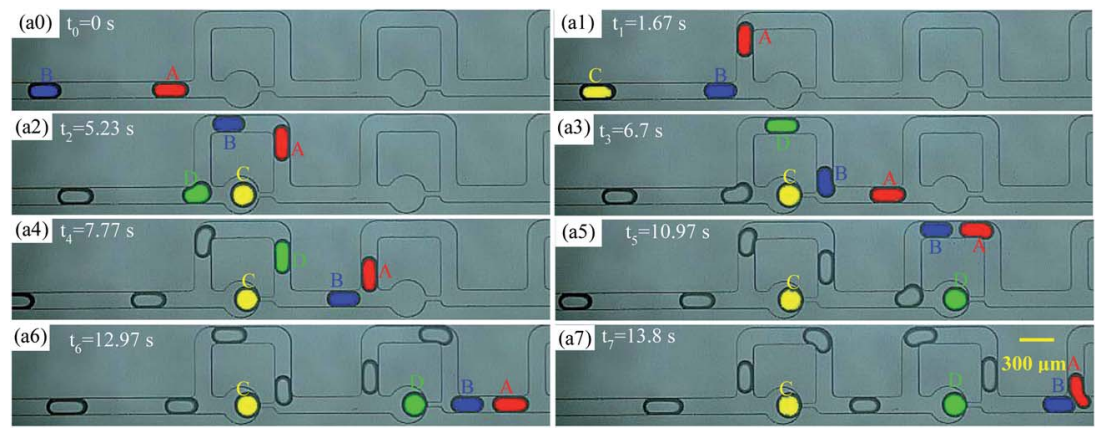

Fig. 6 Paths of droplets by bubble-free guided trapping. The scale bar of all images is $300 \mu \mathrm{m}$ (A, B, C and D represent the first four droplets of the train). 

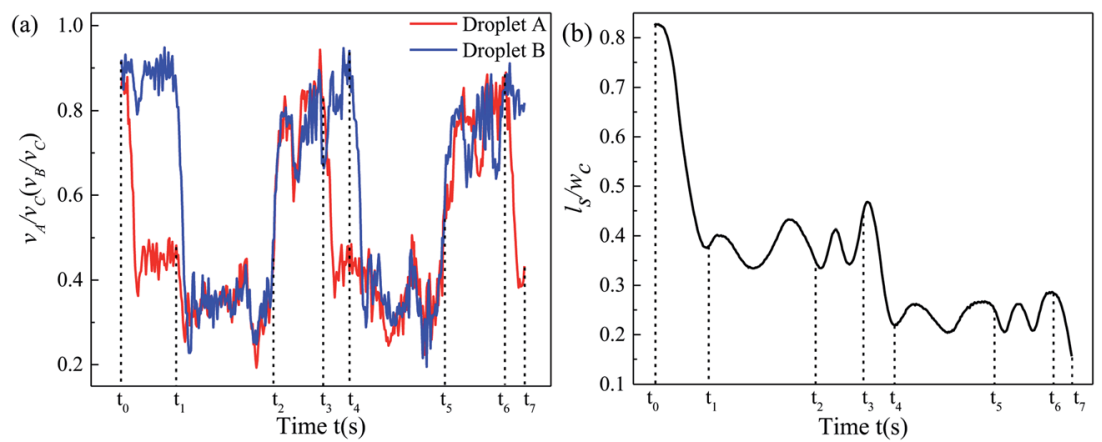

Fig. 7 Variation of the speeds of two neighboring droplets and their spacing. (a) Graph showing how the dimensionless speeds of $A$ and $B$ ( $v_{A} / v_{C}$ and $\left.v_{B} / v_{C}\right)$ vary with time. (b) Graph showing how the dimensionless centroid distance between $A$ and $B\left(I_{s} / w_{c}\right)$ varies with time.

and $\mathrm{T}_{3}$ were observed 15,6 , and 4 times, respectively, in a complete trapping process (see movie 1, ESI $\dagger$ ). When droplets A and B pass through two trapping units (no. 1 and 2), the path selection of the droplets is shown in Fig. 6. The normalized speed of A and B $\left(\alpha=v_{\mathrm{A}} / v_{\mathrm{C}}\right.$ and $\left.\beta=v_{\mathrm{B}} / v_{\mathrm{C}}\right)$, and the normalized centroid distance between A and B $\left(\phi=l_{\mathrm{S}} / w\right)$ over time are shown in Fig. 7(a) and (b), respectively, and $v_{\mathrm{C}}$ is the velocity of the continuous phase in the main channel. The time at which the first droplet enters the first junction of the MTN is marked as $0 \mathrm{~s}$.

The initial spacing of the droplets is heavily disturbed. Specifically, for $t_{0} \sim t_{1}$ (from the time point when the front tip of A just arrives at the junction to the time when the front tip of $B$ arrives), $\alpha$ decreases due to partial oil leakage through the trapping arm. Meanwhile, $\beta$ maintains the original value of the train, and thus B effectively approaches A, and $\phi$ decreases. For $t_{1} \sim t_{2}$ (from the time point when the front tip of $\mathrm{B}$ arrives at the junction to $\mathrm{C}$ being trapped), $\alpha$ is reduced again since $\mathrm{B}$ hampers the addition of oil to the bypass. Additionally, $\alpha$ is slightly increased due to some of the oil in the trapping arm being blocked by $\mathrm{C}$ and more oil being delivered into the bypass. In contrast, for $t_{2} \sim t_{3}$ (from the time point when $\mathrm{C}$ is trapped to the front tip of A arriving at the junction of the next unit), $\alpha$ and $\beta$ approximately recover to the initial value of the train (neglecting the gutter flow in the trapping arm), which is attributed to oil only flowing through the bypass. For $t_{3} \sim t_{6}$, the evolution of $\alpha, \beta$, and $\phi$ over time is analogous to that of $t_{0} \sim t_{3}$.

However, for $t_{6} \sim t_{7}, \phi$ decreases once more such that $\mathrm{A}$ and $\mathrm{B}$ collide. Subsequently, there are three trapping events: (i) B is trapped and A moves through the bypass; (ii) A and B fuse into a long droplet, which fragments into two portions at the junction and one daughter droplet gets trapped, i.e. $\mathrm{T}_{2}$ (see Fig. 4(b)); (iii) the deformation at the junction induced by complex hydrodynamic forces is evidently presented when a larger droplet travels at high speed, resulting in a portion entering the bypass and a portion entering the trapping branch. If the whole body of A does not leave the junction until B catches up, A and B collide and break, behaving as indicated by $\mathrm{T}_{3}$ (see Fig. 4(c)). Thus, the inherent instability of the droplet velocity and the spacing between neighboring droplets is the principal mechanism of the unfavorable events in the MTN.
To address the concerns regarding the shortcoming of the narrow flow rate range of $\mathrm{T}_{1}$ and the non-uniform trapped droplet array, a longer water plug is introduced to fill the microfluidic parking network. Then, oil is injected to shear off the dispersed phase at the junctions in an orderly manner. ${ }^{30} \mathrm{An}$ alternative method was proposed, which utilizes the coalescence between the moving and parked droplets and subsequent break-up to automatically correct the volume of trapped surfactant-free droplets. ${ }^{21}$ Both approaches readily generate a monodisperse droplet array. The former is incompatible with parking droplets in which insoluble materials are encapsulated due to the medium inside the trapped droplets distributing in a random manner..$^{30}$ The latter is not suitable for systems where an excess of surfactant is added (a standard operation in droplet-based microfluidics), because droplet coalescence and volume rectification do not occur in the MTN. Moreover, undesired interfacial mass transfer induced by coalescence heavily hampers applications where each droplet serves as an individual reaction vessel. A bubble-guided trapping method distinct from that of Bithi et al. ${ }^{21}$ is proposed in the present study, which is suitable for both systems in which a surfactant is not added, and systems in which one is added.

\subsection{Mechanism of improved trapping by bubble guidance}

To maintain the original content of each droplet in a train and upgrade the uniformity of the trapped droplets, we can reduce or eliminate the events of coalescence and collision. Thus, a novel approach where an additional bubble guides the droplet train to move through the MTN is proposed. This takes advantage of the hydrodynamic and physical properties of the bubble characterized by a large hydrodynamic resistance, as well as non-coalescence with two-phase liquids.

Likewise, $Q_{\mathrm{d}}=12 \mu \mathrm{L} \mathrm{h}^{-1} Q_{\mathrm{c} 1}=Q_{\mathrm{c} 2}=20 \mu \mathrm{L} \mathrm{h}^{-1}$ is taken as an example to show the mechanism of improved trapping by bubble guidance. The time when the bubble just enters the first junction of the MTN is defined as $0 \mathrm{~s}$. Snapshots of droplets travelling through three units (no. 11 to 13) at six moments are indicated in Fig. 8 to clearly illustrate the trapping mechanism. The droplets $(1,2$, and 3$)$ next to the bubble directly go through the trapping arm and then get parked, effectively avoiding coalescence and collision. The frequency at which $\mathrm{T}_{1}$ occurs and the polydispersity 

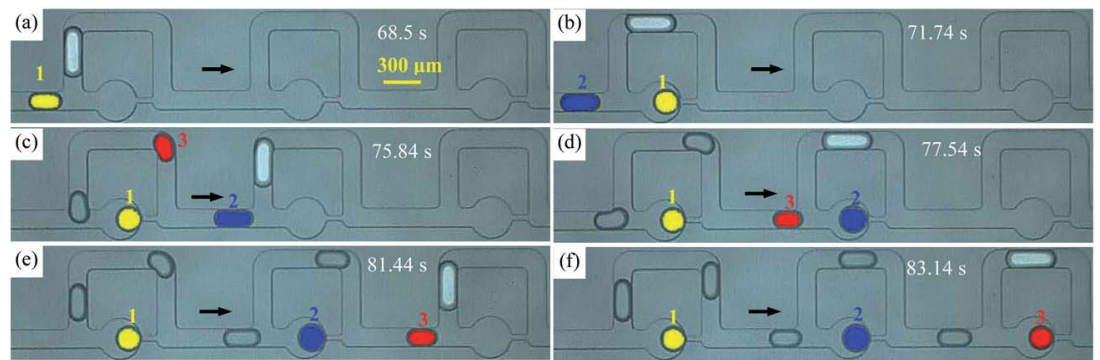

Fig. 8 Droplet trapping by bubble guidance $\left(Q_{d}=12 \mu \mathrm{L} \mathrm{h}^{-1}, Q_{c 1}=20 \mu \mathrm{L} \mathrm{h}^{-1}, Q_{\mathrm{c} 2}=20 \mu \mathrm{L} \mathrm{h}{ }^{-1}\right.$, the arrow indicates the flow direction). (a)-(f) Snapshots of droplets at six critical moments.

are significantly improved. In contrast, the first two droplets of a train always move through the bypass during bubble-free guided trapping and the undesired events as shown in Fig. 6 are readily presented. Thus, bubble guidance makes the droplets more ordered in the MTN compared with those produced by bubble-free guidance, and this is beneficial to expand the flexibility of the MTN and its usability in lab on a chip applications, where mass transfer between reactors is not expected.

According to the qualitative analysis of the path selection of the droplets in Fig. 8, the improvement caused by the bubble is predominantly attributed to the hydrodynamic characteristic of its large resistance at the tested flow rates. All $\mathrm{T}_{1}$ events in Fig. 5(b) follow the rule that the bubble moves along the bypass and the subsequent droplet is trapped, indicating that $Q_{\mathrm{ct}}>Q_{\mathrm{cb}}$, as schematically shown in Fig. 9(a). However, bubble-free guided trapping is different as the first two droplets choose the bypass and the third is trapped, indicating that $Q_{\mathrm{cb}}>Q_{\mathrm{ct}}$, as schematically shown in Fig. 9(b). The trapping effects induced by bubble guidance are equivalent to those induced by twodroplet guidance (assuming that bubble-free guided trapping is guided by droplets). It is roughly inferred that $\Delta P_{\mathrm{B}} \approx 2 \Delta P_{\mathrm{D}}$, where $\Delta P_{\mathrm{B}}$ and $\Delta P_{\mathrm{D}}$ are the pressure drop of the bubble and droplet, respectively. If a droplet whose length is equal to that of a bubble acts as the guider, as shown in Fig. 9 (c1), the two neighboring droplets flow through several units and fuse as illustrated in Fig. 9 (c2). They do not fuse in bubble-guided trapping as shown in Fig. 9 (c3) and (c4) even if the bubble and droplet come into contact. Thus, the advancement is also attributed to the unique physical property that the bubble is immiscible with two-phase fluids.

The resistive feature difference between the two guiding media causes two trapping effects. Therefore, it is necessary to explore the factors that lead to this difference. Wong et al. ${ }^{31}$ gave eqn (2) with the aid of numerical computation to quantify the pressure drop of a bubble in a rectangular channel:

$$
\Delta P=\eta \mathrm{Ca}^{2 / 3}
$$

$\eta$ is a coefficient depending on the channel dimensions and Ca is the capillary number of the continuous phase. The hydrodynamic resistance of a bubble in a rectangular microchannel mainly originates from pressure jumps of the two end caps. ${ }^{32}$ Additionally, Baroud et $a l .^{33}$ pointed out that the inviscid theory for the derivation of bubble pressure drops remains valid for a liquid-liquid microfluidic system where $\mu_{\mathrm{d}} / \mu_{\mathrm{c}}$ (the viscosity ratio of the dispersed to continuous phase) is far less than unity. $\mu_{\mathrm{d}} / \mu_{\mathrm{c}}$ is equal to 0.018 in the present liquid-liquid system. The pressure drop across the bubble and droplet can be calculated using eqn (2) in the present study. It is noted that the effect of bends in the bypass channel is ignored in the discussion. Here, we only give a reasonably qualitative analysis of the pressure drop difference between the bubble and the droplet to illustrate the main mechanism for the improvement of the trapping effect. Thus, the difference in hydrodynamic resistance is mainly due to the difference in interfacial tension. The interfacial tensions measured using a rheometer are $\gamma_{1}=25.3 \mathrm{mN}$ $\mathrm{m}^{-1}$ between water and oil, and $\gamma_{2}=49.1 \mathrm{mN} \mathrm{m}^{-1}$ between air and oil in our experiments. $\Delta P_{\mathrm{B}} / \Delta P_{\mathrm{D}}=\sqrt[3]{\left(\gamma_{2} / \gamma_{1}\right)^{2}}=1.56>1$ agrees well with that deduced from the law of droplet path selection (Fig. 9(a) and (b)). Thus, the desired trapping induced by bubble guidance is mainly attributed to the large hydrodynamic resistance compared with bubble-free guided trapping.

The hydrodynamic resistance of a bubble or droplet in a straight or curved microchannel is a complex and fundamental research aspect and related to numerous factors, including flow velocity, spacing, and viscosity. ${ }^{20,34-36}$ Existing studies are focused on the qualitative analysis and indirect measurement of straight

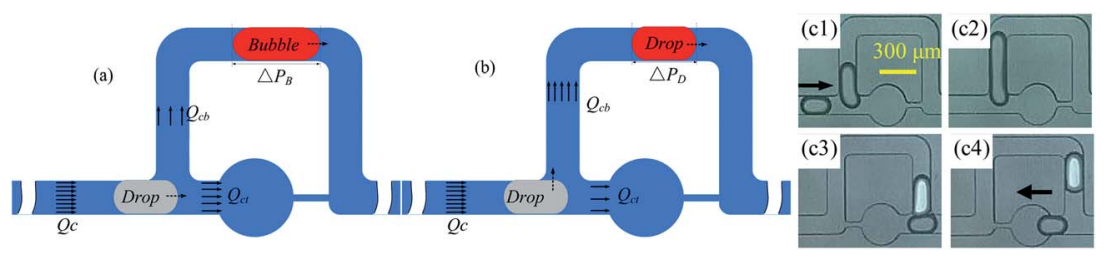

Fig. 9 Schematic diagram of the oil flow distribution in a trapping unit; the dotted arrow indicates the movement direction of the droplet. (a) Oil distribution in a trapping unit by bubble guidance. (b) Oil distribution in a trapping unit by bubble-free guidance. (c) Comparison of bubble-free and bubble-guided trapping; the arrow indicates the flow direction. 
microchannels; it is challenging to quantitatively measure and compare their hydrodynamic resistance under the same conditions and further research is needed.

\section{Conclusions}

The trapping modes of a moving droplet train by bubble-free and bubble guidance are comparatively studied in this paper. For bubble-free guided trapping, disordered non-uniform trapping induced by break-up and collision is observed in a wide range and heavily attributed to the intrinsic instability of droplet spacing in the microfluidic trapping network. In contrast, the flow region of sequentially uniform trapping is significantly broadened by bubble-guided trapping and this is predominantly attributed to the large hydrodynamic resistance of the bubble.

The bubble-guided trapping method also makes significant progress in addressing the concerns regarding reagent nonuniformity in the network, and is especially suitable for applications (e.g. the observation of Caenorhabditis elegans) where mass transfer between micro-reactors should be avoided. Although the size of the bubble is not accurate due to empirical control, the highlighted results provide an ideal scenario and guidelines to improve the performance and suitability of microfluidic chips for passive micro-reactor storage.

\section{Conflicts of interest}

There are no conflicts to declare.

\section{Acknowledgements}

The authors gratefully acknowledge the support of the National Natural Science Foundation of China (Grant No. 11572013, and No. 11702007).

\section{References}

1 Y. Ding, J. Choo and A. J. deMello, Microfluid. Nanofluid., 2017, 21, 58.

2 Y. Piao, D. J. Han, M. R. Azad, M. Park and T. S. Seo, Biosens. Bioelectron., 2015, 65, 220-225.

3 M. Courtney, X. Chen, S. Chan, T. Mohamed, P. P. N. Rao and C. L. Ren, Anal. Chem., 2017, 89, 910-915.

4 S. Ralf, B. Martin, P. Thomas and H. Stephan, Rep. Prog. Phys., 2012, 75, 016601.

5 L. Zhang, G. Niu, N. Lu, J. Wang, L. Tong, L. Wang, M. J. Kim and Y. Xia, Nano Lett., 2014, 14, 6626-6631.

6 K. S. McMillan, M. Boyd and M. Zagnoni, Lab Chip, 2016, 16, 3548-3557.

7 M. Srisa-Art, A. J. deMello and J. B. Edel, J. Phys. Chem. B, 2010, 114, 15766-15772.

8 H.-H. Jeong, B. Lee, S. H. Jin, S.-G. Jeong and C.-S. Lee, Lab Chip, 2016, 16, 1698-1707.
9 S. H. Jin, H.-H. Jeong, B. Lee, S. S. Lee and C.-S. Lee, Lab Chip, 2015, 15, 3677-3686.

10 R. W. Rambach, K. Linder, M. Heymann and T. Franke, Lab Chip, 2017, 17, 3422-3430.

11 H. Boukellal, S. Selimovic, Y. Jia, G. Cristobal and S. Fraden, Lab Chip, 2009, 9, 331-338.

12 W. Shi, J. Qin, N. Ye and B. Lin, Lab Chip, 2008, 8, 1432.

13 S. S. Bithi and S. A. Vanapalli, Biomicrofluidics, 2010, 4, 044110.

14 M. G. Simon, R. Lin, J. S. Fisher and A. P. Lee, Biomicrofluidics, 2012, 6, 014110.

15 G. Amselem, P. T. Brun, F. Gallaire and C. N. Baroud, Phys. Rev. Appl., 2015, 3, 054006.

16 W. Shi, H. Wen, Y. Lu, Y. Shi, B. Lin and J. Qin, Lab Chip, 2010, 10, 2855-2863.

17 W. H. Tan and S. Takeuchi, Proc. Natl. Acad. Sci. U. S. A., 2007, 104, 1146-1151.

18 S. S. Bithi and S. A. Vanapalli, Sci. Rep., 2017, 7, 41707.

19 X. Chen and C. Ren, RSC Adv., 2017, 7, 16738-16750.

20 V. Labrot, M. Schindler, P. Guillot, A. Colin and M. Joanicot, Biomicrofluidics, 2009, 3, 012804.

21 S. S. Bithi and S. A. Vanapalli, Soft Matter, 2015, 11, 51225132 .

22 S. S. Bithi, W. S. Wang, M. Sun, J. Blawzdziewicz and S. A. Vanapalli, Biomicrofluidics, 2014, 8, 034118.

23 W.-C. Cheng, Y. He, A.-Y. Chang and L. Que, Biomicrofluidics, 2013, 7, 064102.

24 H.-H. Jeong, S. H. Jin, B. J. Lee, T. Kim and C.-S. Lee, Lab Chip, 2015, 15, 889-899.

25 A. Dewan, J. Kim, R. H. McLean, S. A. Vanapalli and M. N. Karim, Biotechnol. Bioeng., 2012, 109, 2987-2996.

26 W.-A. C. Bauer, M. Fischlechner, C. Abell and W. T. S. Huck, Lab Chip, 2010, 10, 1814-1819.

27 H. Boukellal, S. Selimovic, Y. Jia, G. Cristobal and S. Fraden, Lab Chip, 2009, 9, 331-338.

28 M. Sun, S. S. Bithi and S. A. Vanapalli, Lab Chip, 2011, 11, 3949-3952.

29 W. Shi, J. Qin, N. Ye and B. Lin, Lab Chip, 2008, 8, 1432-1435.

30 M. Sun, S. S. Bithi and S. A. Vanapalli, Lab Chip, 2011, 11, 3949-3952.

31 H. Wong, C. J. Radke and S. Morris, J. Fluid Mech., 1995, 292, 95-110.

32 P. Parthiban and S. A. Khan, Lab Chip, 2012, 12, 582-588.

33 C. N. Baroud, F. Gallaire and R. Dangla, Lab Chip, 2010, 10, 2032-2045.

34 S. A. Vanapalli, A. G. Banpurkar, D. van den Ende, M. H. G. Duits and F. Mugele, Lab Chip, 2009, 9, 982-990.

35 M. J. Fuerstman, A. Lai, M. E. Thurlow, S. S. Shevkoplyas, H. A. Stone and G. M. Whitesides, Lab Chip, 2007, 7, 14791489.

36 P. Sajeesh, M. Doble and A. K. Sen, Biomicrofluidics, 2014, 8, 054112. 